\title{
Enlighten e-cigarettes
}

\author{
The use of electronic cigarettes is on the rise, but we need more evidence about their risks as well as their value in \\ curbing tobacco use. Ultimately, we need to design better, smarter vaping tools for smoking cessation.
}

ate last year, the US government released
new statistics on electronic cigarettes
that shocked public health officials: the number of high school students in the country who reported that they used e-cigarettes jumped nearly $80 \%$ from 2017 to 2018 . One in five teens regularly engage in vaping, according to the findings of the report from the US Food and Drug Administration (FDA) and Centers for Disease Control and Prevention. It's no surprise then that the American Lung Association gave the FDA an 'F' grade in January for failing to protect children from e-cigarettes. A study published the following month found that teens who try e-cigarettes are far more likely to end up smoking tobacco cigarettes than those who have never vaped (JAMA Netw. Open. 2, e187794, 2019). Meanwhile, producers of these devices continue to make record sales: the e-cigarette maker Juul reportedly had more than $\$ 1$ billion in sales in 2018, five times the amount of the previous year.

E-cigarettes were widely touted as the solution to help smokers quit traditional tobacco cigarettes. And recent data have shown that this approach has promise. In February, the New England Journal of Medicine published a study in which adults seeking to quit smoking through the programs run by the UK's National Health Service were randomly assigned e-cigarettes or other nicotine replacement products. At the one-year follow-up, $18 \%$ of those who received e-cigarettes were abstaining from smoking traditional cigarettes, compared with less than $10 \%$ of those who were given other types of nicotine replacement options (N. Engl. J. Med. 380, 629-637, 2019).

But e-cigarettes are far from a panacea. In fact, some smoking cessation programs have expanded in recent years to help people quit e-cigarettes as well. Former e-cigarette users have called the process of quitting these devices 'hell', and health officials worry that the concentrated nicotine in these products only makes them more addictive. Another February study concluded that there is a 'nicotine arms race. "Juul has triggered a widespread rush among aerosol purveyors to market e-liquid in unprecedentedly high nicotine concentrations," the authors wrote (Tob. Control https://doi.org/10.1136/ tobaccocontrol-2018-054796, 2019).

Given the addictive nature of nicotine and potent effects on biochemical pathways in the body, scientists have sought to understand whether it is carcinogenic. Studies have found evidence that nicotine can enhance the carcinogenesis of malignant cancer cells inoculated in mice. Scientists note that this is particularly true in immunocompromised mice. The authors of a review on this matter concluded that, "Overall, taking the human and animal studies into consideration, there appears to be inadequate evidence to conclude that nicotine per se does or does not cause or modulate carcinogenesis in humans" (Crit. Rev. Toxicol. 46, 701-734, 2016).

Notably, studies of Swedish users of snus (a moist powder tobacco product placed in the upper lip), who obtain nicotine levels similar to smokers, did not find associations between nicotine and cancer.

There is some evidence that nicotine might contribute to acute cardiovascular events in individuals with underlying coronary heart disease, but we need to look beyond just nicotine when assessing the risk of e-cigarettes. At the American Stroke Association's International Stroke Conference in Honolulu in February, data presented from a study of 400,000 individuals revealed that e-cigarette users were at heightened risk of heart attack and stroke, although the scientists behind the research note that many e-cigarette users were also twice as likely to smoke regular cigarettes, which could be a confounding factor for these results. At the same time, trauma centers have seen a number of instances of people with burn injuries from exploding e-cigarettes.

Healthcare researchers should ask developers to consider making safer and more effective e-cigarettes with features more attuned to facilitation of smoking cessation. There is some innovation in this space already. Devices like the Enovap Personal Vaporizer allow users to control the nicotine levels in the product via a Bluetooth connection to monitor intake from e-cigarettes, enabling users to work toward a goal to cut down. But perhaps there is a role for more physician involvement. In 2014, the start-up Thermo-Essence Technologies announced that it would develop a prescription-only e-cigarette that would dispense physician-determined doses of nicotine each day and would then send usage data of the device back to the doctor. The company, however, has folded, and this idea never came to fruition. Instead, the FDA has regulated e-cigarettes like other tobacco products, rather than as a medical device. Perhaps it is time to rethink this classification of e-cigarettes and regulate them more closely.

It's not inconceivable that the medical aims of e-cigarettes are at odds with the business aims of their manufacturers. The global e-cigarette industry was a \$14 billion industry in 2017, and one can assume that the numbers have only risen since then. It is in the interest of companies that make vaping devices to keep consumers addicted to their products. The global health community needs to recognize this possible conflict of interest among e-cigarette makers and apply more stringent rules to how these products are sold and used. E-cigarettes can be useful tools in the war against tobacco, but only if wielded wisely.

Published online: 4 April 2019 https://oi.org/10.1038/s41591-019-0431-5 